\title{
The Bound-state Soliton Solutions of a Higher-order Nonlinear Schrödinger Equation for Inhomogeneous Heisenberg Ferromagnetic System
}

\section{Jin-Jin Mao}

Beijing Institute of Technology

Shoufu Tian ( $\square$ sftianmath@126.com )

China University of Mining and Technology

Tian-Zhou Xu

Beijing Institute of Technology

Lin-Fei Shi

Beijing Institute of Technology

\section{Research Article}

Keywords: A higher-order nonlinear Schr“ odinger equation ,Heisenberg ferromagnetism system ,RiemannHilbert problem ,Initial value problem ,Multiple higher-order poles·Bound-state soliton solution

Posted Date: February 18th, 2021

DOl: https://doi.org/10.21203/rs.3.rs-207395/v1

License: (c) (i) This work is licensed under a Creative Commons Attribution 4.0 International License. Read Full License

Version of Record: A version of this preprint was published at Nonlinear Dynamics on April 15th, 2021. See the published version at https://doi.org/10.1007/s11071-021-06425-z. 


\title{
The bound-state soliton solutions of a higher-order nonlinear Schrödinger equation for inhomogeneous Heisenberg ferromagnetic system
}

\author{
Jin-Jin Mao* • Shou-Fu Tian* • Tian-Zhou \\ Xu • Lin-Fei Shi
}

Received: date / Accepted: date

\begin{abstract}
By studying an appropriate Riemann-Hilbert (RH) problem, the inverse scattering of a higher-order nonlinear Schrödinger equation for inhomogeneous Heisenberg ferromagnetic system with zero boundary condition is calculated. The RH problem of reflection coefficient with multiple high-order poles is obtained. Meanwhile, the calculation formulas of bound state (BS) solitons and multiple BS solitons corresponding to one high-order pole and multiple high-order poles are also calculated respectively. Finally, the corresponding soliton solution model is calculated according to the corresponding formula. Simultaneously, we also obtain the interaction between BS soliton and multiple BS solitons, and between multiple BS solitons and multiple BS solitons.
\end{abstract}

Keywords A higher-order nonlinear Schrödinger equation - Heisenberg ferromagnetism system · Riemann-Hilbert problem · Initial value problem · Multiple higher-order poles $\cdot$ Bound-state soliton solution.

Tian-Zhou Xu $\cdot$ Lin-Fei Sh

School of Mathematics and Statistics, Beijing Institute of Technology, Beijing 100081, People's Republic of China

Jin-Jin Mao ( $\otimes)$ (Corresponding author)

School of Mathematics and Statistics, Beijing Institute of Technology, Beijing 100081, People's Republic of China

E-mail: 3120205703@bit.edu.cn

Shou-Fu Tian $(\otimes)$ (Corresponding author)

School of Mathematics and Institute of Mathematical Physics, China University of Mining and Technology, Xuzhou 221116, People's Republic of China

E-mail: sftian@cumt.edu.cn, sftianmath@126.com

*Corresponding author: sftian@cumt.edu.cn, shoufu2006@126.com (Shou-Fu Tian) 


\section{Introduction}

In recent years, the Heisenberg magnetic spin chain has drawn much attention[1][5] because one-dimensional spin array can store quantum information[2]. At the same time, it is found that the Heisenberg ferromagnetic (HBF) models with different magnetic interactions are equivalent to a class of nonlinear evolution equations in semi-classical and continuous media[6-9].

Besides, an inhomogeneous HBF spin system with extended structure is also obtained [10]

$$
\begin{aligned}
\vec{T}_{t}= & \frac{1}{2} \vec{T} \times \vec{T}_{x x}+h \vec{T}_{x}+\frac{5}{2} \epsilon\left(\vec{T}_{x} \cdot \vec{T}_{x}\right) \vec{T}_{x x x}+10 \epsilon\left(\vec{T}_{x} \cdot \vec{T}_{x x}\right) \vec{T}_{x x}+\epsilon \vec{T}_{x x x x x} \\
& +\epsilon\left[\frac{35}{2}\left(\vec{T}_{x} \cdot \vec{T}_{x}\right)\left(\vec{T}_{x} \cdot \vec{T}_{x x}\right)+10 \vec{T}_{x x} \vec{T}_{x x x}+5 \vec{T}_{x} \vec{T}_{x x x x}\right] \vec{T} \\
& +\epsilon\left[\frac{35}{8}\left(\vec{T}_{x} \cdot \vec{T}_{x}\right)^{2}+10 \vec{T}_{x} \cdot \vec{T}_{x x x}+\frac{15}{2} \vec{T}_{x x} \cdot \vec{T}_{x x}\right] \vec{T}_{x},
\end{aligned}
$$

where $\epsilon$ means the perturbation parameter, $h$ is the inhomogeneity of the medium, and $\vec{T}$ represents the spin vector relative to the space coordinate $x$ and the scale time $t$. It is found that equation (1) can degenerate into the HBF spin system at $\epsilon=h=0$.

In addition, the soliton solutions of some nonlinear equations have been solved, such as the nonlinear Schrödinger (NLS) equation

$$
i \Phi_{t}+\Phi^{2} \Phi^{*}+\frac{1}{2} \Phi_{x x}=0
$$

where the superscript $*$ denotes the complex conjugate, and $\Phi$ is a function of $x$ and $t$. It has very significant physical significance in fluid mechanics, Bose Einstein condensation, nonlinear optics and so on. For example, it can describe the group evolution process of quasi monochromatic waves in weakly nonlinear dispersive media. Because of its crucial physical significance, NLS equation has attracted extensive attention, especially after Zakharov and shabat et al [11]-[12].

In this paper, we study the higher-order NLS equation equivalent to equation (1) by mapping the spin vector function $\vec{T}$ to the unit tangent vector of the motion helical space curve[13], which has the following form [14]

$$
\begin{gathered}
i q_{t}+q^{2} q^{*}-30 i \epsilon q_{x} q^{2}\left(q^{*}\right)^{2}-10 i \epsilon q_{x x} q q_{x}^{*}-10 i \epsilon q_{x} q q_{x x}^{*}-10 i \epsilon q_{x x} q q^{*} \\
-10 i \epsilon q_{x}^{2} q_{x}^{*}-20 i \epsilon q_{x} q_{x x} q^{*}+\frac{1}{2} q_{x x}-i h q_{x}-i \epsilon q=0
\end{gathered}
$$

where $q$ is a function of $x$ and $t$. The above equation is the compatibility condition of the following Lax pairs

$$
\frac{\partial}{\partial x} \phi(x, t, \lambda)=U \phi(x, t, \lambda), \quad \frac{\partial}{\partial t} \phi(x, t, \lambda)=V \phi(x, t, \lambda),
$$


where $\phi(x, t, \lambda)$ is a $2 \times 2$ matrix function, $U$ and $V$ are expressed in the following form, respectively

$$
\begin{aligned}
U= & -i \lambda \sigma_{3}+Q, \\
V= & \frac{1}{2}\left[-2 i\left(h \lambda+\lambda^{2}+16 \epsilon \lambda^{5}\right) \sigma_{3}-i\left(1+16 \epsilon \lambda^{3}\right) Q^{2} \sigma_{3}-12 i \epsilon \lambda Q^{4} \sigma_{3}+8 \epsilon \lambda^{2}\left(Q_{x} Q-Q Q_{x}\right)\right. \\
& +12 \epsilon Q^{2}\left(Q_{x} Q-Q Q_{x}\right)-4 i \epsilon \lambda Q_{x}^{2} \sigma_{3}+4 i \epsilon \lambda\left(Q_{x x} Q+Q Q_{x x}\right) \sigma_{3}+2 h Q+2 \lambda Q \\
& +2 \epsilon\left(Q Q_{x x x}-Q_{x x x} Q\right)-2 \epsilon\left(Q_{x} Q_{x x}-Q_{x x} Q_{x}\right)+32 \epsilon \lambda^{4} Q+16 \epsilon \lambda^{2} Q^{3}+12 \epsilon Q^{5} \\
& +16 i \epsilon \lambda^{3} Q_{x} \sigma_{3}-24 i \epsilon \lambda Q^{2} Q_{x} \sigma_{3}-12 \epsilon Q^{*} Q_{x}^{*} Q_{x}-8 \epsilon Q Q_{x}^{2}-8 \epsilon \lambda^{2} Q_{x x}-16 \epsilon Q^{2} Q_{x x} \\
& \left.-4 \epsilon Q Q^{*} Q_{x x}^{*}+4 i \epsilon \lambda Q_{x x x} \sigma_{3}+2 \epsilon Q_{x x x x}-i Q_{x} \sigma_{3}\right],
\end{aligned}
$$

with

$$
Q=\left(\begin{array}{cc}
0 & q \\
-q^{*} & 0
\end{array}\right), \quad \sigma_{3}=\left(\begin{array}{cc}
1 & 0 \\
0 & -1
\end{array}\right)
$$

In [14], Jia and Ma worked out the higher-order rogue wave solutions of equation (3) and analyzed their properties by the method of generalized Darboux transformation. We also note that when we make $\epsilon=h=0$ in equation (3), we can reduce equation (3) to equation (2). In [15], the three parameter two period solution family of NLS equation (2), the repetition, period doubling and amplification outside the conventional modulation instability band are studied in detail.

The inverse scattering transformation (IST) is one of the effective methods for solving nonlinear integrable equations. IST was first proposed by Gardner, Green and others, and IST purpose is to study the initial value problem of the KdV equation $[16,17]$. After that, there are many works extended IST to other equations [18][27]. Based on the asymptotic behavior of the Jost solution, the Gelfand-LevitanMarchenko (GLM) integral equation is constructed, and soliton of the KdV equation is represented by solution of the GLM equation. In recent years, a new IST for focusing on the NLS equation has been proposed $[28,29]$, which is an improvement on the standard IST and is very helpful in dealing with the equations with serious spectral singularities. For example, it is difficult to find the rogue wave solution of linear integrable equation with standard IST, but this new method can be used to solve it (Ref. [28] obtained rogue wave solution of the NLS equation by using new IST).

The structure of this paper is as follows. In section 2, we first construct the Riemann-Hilbert (RH) problem of equation (3), and then show the relationship between the solution of RH problem and the solution of equation (3). In section 3, we derive the bound state (BS) soliton and multiple BS soliton solutions of equation (3). In section 4 , we give some explicit soliton solutions and corresponding dynamical properties. In the last section, we give the conclusion. 


\section{The construction of Riemann-Hilbert problem}

2.1 Spectral analysis

In Lax pair (4), if $\phi$ is a $2 \times 2$ matrix, we can obtain that the solution of equation (3) satisfies the symmetric condition

$$
\phi(x, t, \lambda)=\sigma_{0} \phi^{*}\left(x, t, \lambda^{*}\right) \sigma_{0}^{-1},
$$

where $\sigma_{0}=\left(\begin{array}{cc}0 & 1 \\ -1 & 0\end{array}\right)$. Then, we find that when $\phi(x, t, \lambda)$ is a solution to equation (3), $\sigma_{0} \phi^{*}(x, t, \lambda) \sigma_{0}^{-1}$ is also a solution to equation (3). Simultaneously, we can also use this symmetry condition to define the scattering matrix $S(\lambda)$ and solve the RH problem.

We introduce the following hypothesis

$$
\phi(x, t, \lambda)=\psi(x, t, \lambda) e^{-i\left[\lambda x+\left(h \lambda+\lambda^{2}+16 \epsilon \lambda^{5}\right) t\right] \sigma_{3}},
$$

we consider both $x$-part and $t$-part of the Lax pair (4), subsequently. Thus we can obtain the relationship of the Lax pair $\psi(x, t, \lambda)$ is

$$
\begin{aligned}
& \psi_{x}+i \lambda\left[\sigma_{3}, \psi\right]=Q \psi, \\
& \psi_{t}+i\left(h \lambda+\lambda^{2}+16 \epsilon \lambda^{5}\right)\left[\sigma_{3}, \psi\right]=\widetilde{Q} \psi,
\end{aligned}
$$

with

$$
\begin{aligned}
\widetilde{Q}= & -i\left(\frac{1}{2}+8 \epsilon \lambda^{3}\right) Q^{2} \sigma_{3}-6 i \epsilon \lambda Q^{4} \sigma_{3}+4 \epsilon \lambda^{2}\left(Q_{x} Q-Q Q_{x}\right)+6 \epsilon Q^{2}\left(Q_{x} Q-Q Q_{x}\right) \\
& -2 i \epsilon \lambda Q_{x}^{2} \sigma_{3}+2 i \epsilon \lambda\left(Q^{*} Q_{x x}+Q Q_{x x}\right) \sigma_{3}+\epsilon\left(Q_{x}^{*} Q_{x x}-Q_{x} Q_{x x}\right)+\epsilon\left(Q Q_{x x x}-Q^{*} Q_{x x x}^{*}\right) \\
& +h Q+2 \lambda Q+16 \epsilon \lambda^{4} Q+8 \epsilon \lambda^{2} Q^{3}+6 \epsilon Q^{5}-\frac{i}{x} Q_{x} \sigma_{3}-8 i \epsilon \lambda^{3} Q_{x} \sigma_{3}-12 i \epsilon \lambda Q^{2} Q_{x} \sigma_{3} \\
& -6 \epsilon Q^{*} Q_{x}^{*} Q_{x}-4 \epsilon Q Q_{x}^{2}-4 \epsilon \lambda^{2} Q_{x x}-8 \epsilon Q^{2} Q_{x x}-2 \epsilon Q Q^{*} Q_{x x}^{*}+2 i \epsilon \lambda Q_{x x x} \sigma_{3}+\epsilon Q_{x x x x} .
\end{aligned}
$$

Subsequently, we will discuss the case of rapidly decay initial conditions

$$
q(x, 0)=q_{0}(x), \quad \text { and } \quad q_{0}(x) \rightarrow 0 \quad \text { as } \quad x \rightarrow \infty,
$$

and then asymptotic solution $\psi(x, t, \lambda)$ will satisfy

$$
\psi_{ \pm}(x, t, \lambda) \rightarrow I, \quad x \rightarrow \pm \infty .
$$

The lax pair of $\psi(x, t, \lambda)$ is formally integrable, and its Jost solutions can be expressed in the following Volterra integral form

$$
\psi_{ \pm}(x, t, \lambda)=I+\int_{ \pm \infty}^{x} e^{-i \lambda(x-y) \sigma_{3}} Q(y, t) \psi_{ \pm}(x, t, \lambda) e^{i \lambda(x-y) \sigma_{3}} d y
$$

Proposition 2.1. The Jost solution $\psi_{ \pm}(x, t, \lambda)$ has the following analytic properties

(I) $\left[\psi_{-}\right]_{1},\left[\psi_{+}\right]_{2}$ are analytic in $\{\lambda \mid \operatorname{Im} \lambda>0\}$ and continuous in $\{\lambda \mid \operatorname{Im} \lambda \geq 0\}$; 
(II) $\left[\psi_{+}\right]_{1},\left[\psi_{-}\right]_{2}$ are analytic in $\{\lambda \mid \operatorname{Im} \lambda<0\}$ and continuous in $\{\lambda \mid \operatorname{Im} \lambda \leq 0\}$,

and its also has the following asymptotic property

(i) $\left[\left[\psi_{-}\right]_{1},\left[\psi_{+}\right]_{2}\right] \rightarrow I$ as $\lambda \rightarrow \pm \infty$ in $\{\lambda \mid \operatorname{Im} \lambda>0\}$;

(ii) $\left[\left[\psi_{+}\right]_{1},\left[\psi_{-}\right]_{2}\right] \rightarrow I$ as $\lambda \rightarrow \pm \infty$ in $\{\lambda \mid \operatorname{Im} \lambda<0\}$,

where we need to explain that $\left[\psi_{ \pm}\right]_{1},\left[\psi_{ \pm}\right]_{2}$ represents the 1-th column and the 2-th column of matrix $\left[\psi_{ \pm}\right]$, respectively. In addition, the solution of equation (3) can be expressed as follows

$$
q(x, t)=\lim _{x \rightarrow \infty}\left[2 i \lambda\left(\psi_{ \pm}\right)_{12}\right],
$$

where $\left(\psi_{ \pm}\right)_{12}$ represent the 1-th row and the 2-th column of matrix $\psi_{ \pm}$.

Proof. For the proof of the above proposition, we can refer to Ref. [30,31].

According to Abel's theorem, any solution $\phi(x, t, \lambda)$ of (4) satisfies $\partial_{x}(\operatorname{det} \phi)=$ $\partial_{t}(\operatorname{det} \phi)=0$. Due to $\lim _{x \rightarrow \pm \infty} \phi(x, t, \lambda)=e^{-i\left[\lambda x+\left(h \lambda+\lambda^{2}+16 \epsilon \lambda^{5}\right) t\right] \sigma_{3}}$, we can have

$$
\operatorname{det} \phi_{ \pm}(x, t, \lambda)=1, \quad x, t \in \mathbb{R},
$$

and $\operatorname{det} \phi_{+}(x, t, \lambda)$ and $\operatorname{det} \phi_{-}(x, t, \lambda)$ are linearly correlated. Where $\operatorname{det} \phi_{+}(x, t, \lambda)$ and $\operatorname{det} \phi_{-}(x, t, \lambda)$ represent the solution of scattering problem (4), and they have different asymptotic properties. Let

$$
\phi_{+}(x, t, \lambda)=\phi_{-}(x, t, \lambda) S(\lambda),
$$

or

$$
\psi_{+}(x, t, \lambda)=\psi_{-}(x, t, \lambda) e^{-i\left[\lambda x+\left(h \lambda+\lambda^{2}+16 \epsilon \lambda^{5}\right) t\right] \sigma_{3}} S(\lambda) e^{i\left[\lambda x+\left(h \lambda+\lambda^{2}+16 \epsilon \lambda^{5}\right) t\right] \sigma_{3}},
$$

where $S(\lambda)$ is often referred to as the scattering matrix. By using the symmetry condition given in (7), we obtain

$$
S(\lambda)=\left(\begin{array}{cc}
c^{*}\left(\lambda^{*}\right) & d(\lambda) \\
-d^{*}\left(\lambda^{*}\right) & c(\lambda)
\end{array}\right)
$$

$c(\lambda)$ and $d(\lambda)$ are often referred to as the scattering date. Moreover, on the basis of (17) and (18), we can get

$$
S(\lambda)=e^{i\left[\lambda x+\left(h \lambda+\lambda^{2}+16 \epsilon \lambda^{5}\right) t\right] \sigma_{3}} \psi_{-}^{-1}(x, t, \lambda) \psi_{+}(x, t, \lambda) e^{-i\left[\lambda x+\left(h \lambda+\lambda^{2}+16 \epsilon \lambda^{5}\right) t\right] \sigma_{3}},
$$

or

$$
c(\lambda)=\operatorname{det}\left(\left[\psi_{-}\right]_{1},\left[\psi_{+}\right]_{2}\right), \quad d(\lambda)=e^{2 i\left[\lambda x+\left(h \lambda+\lambda^{2}+16 \epsilon \lambda^{5}\right) t\right] \sigma_{3}} \operatorname{det}\left(\left[\psi_{+}\right]_{2},\left[\psi_{-}\right]_{2}\right) .
$$

From the above formula, the scattered data $c(\lambda)$ and $d(\lambda)$ can be represented by the initial data $q_{0}(x)$. However, given the initial data $q_{0}(x)$, we can get the Jost solution $\psi_{ \pm}(x, t, \lambda)$, then $c(\lambda)$ and $d(\lambda)$ can be calculated by using equations (13) and (20).

According to the analytic analysis of Jost solutions $\psi_{ \pm}(x, t, \lambda)$ in property 2.1 , we derive the following properties

Proposition 2.2. The scattering date $c(\lambda)$ and $d(\lambda)$ satisfy

(i) $|c(\lambda)|^{2}+|d(\lambda)|^{2}=1$ for $\lambda \in \mathbb{R}$;

(ii) $c(\lambda) \rightarrow 1$ as $\lambda \rightarrow \infty ; d(\lambda) \rightarrow 0$ as $\lambda \rightarrow \infty$;

(iii) $c(\lambda)$ is analytic in $\{\lambda \mid \operatorname{Im} \lambda>0\}$ and continuous in $\{\lambda \mid \operatorname{Im} \lambda \geq 0\}$; $d(\lambda)$ is continuous on the real $\lambda$. 
2.2 The Riemann-Hilbert problem

Reviewing the analytical properties of $\psi_{ \pm}(x, t, \lambda)$ in property 2.1 , we rewrite the scattering relation (17) into a piecewise meromorphic $2 \times 2$ matrix function. $\mathbf{M}(x, t, \lambda)$ is defined as

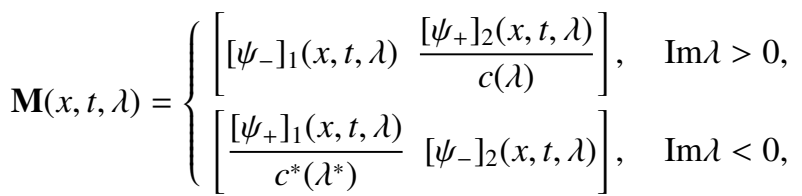

and

$$
\mathbf{M}_{ \pm}(x, t, \lambda)=\lim _{\varepsilon \rightarrow 0+} \mathbf{M}(x, t, \lambda \pm i \varepsilon), \quad \varepsilon, \lambda \in \mathbb{R} .
$$

According to the scattering relation (17), the limit value $\mathbf{M}_{ \pm}(x, t, \lambda)$ satisfies the following conditions

$$
\mathbf{M}_{+}(x, t, \lambda)=\mathbf{M}_{-}(x, t, \lambda) e^{-i\left[\lambda x+\left(h \lambda+\lambda^{2}+16 \epsilon \lambda^{5}\right) t\right] \sigma_{3}} \mathcal{J}(\lambda) e^{i\left[\lambda x+\left(h \lambda+\lambda^{2}+16 \epsilon \lambda^{5}\right) t\right] \sigma_{3}}, \quad \lambda \in \mathbb{R},
$$

and jump matrix $\mathcal{J}(\lambda)$ is

$$
\mathcal{J}(\lambda)=\left[\begin{array}{cc}
1 & r(\lambda) \\
r^{*}\left(\lambda^{*}\right) & 1+|r(\lambda)|^{2}
\end{array}\right] .
$$

with $r(\lambda)=\frac{d(\lambda)}{c(\lambda)}$.

Proposition 2.3. According to the definition of $\mathbf{M}(x, t, \lambda)$, the $\mathrm{RH}$ problem satisfies the following properties

(i) $\operatorname{det} \mathbf{M}=1$;

(ii)Normalization: $\mathbf{M}(x, t, \lambda) \rightarrow I$ as $\lambda \rightarrow \infty$;

(iii)Symmetry

$$
\mathbf{M}^{*}\left(x, t, \lambda^{*}\right)=\sigma_{0} \mathbf{M}(x, t, \lambda) \sigma_{0}^{-1} .
$$

In addition, on the basis of expression (14), we can use $\mathbf{M}(x, t, \lambda)$ to reconstruct the new solution of equation (3) as follows

$$
q(x, t)=\lim _{\lambda \rightarrow \infty} 2 i \lambda \mathbf{M}_{12}(x, t, \lambda) .
$$

If $c(\lambda) \neq 0$ for all $\lambda$, we can get that the RH problems constructed by (21) is regular and can uniquely be solved by Plemelj formula [32]. If $c(\lambda)=0$ at certain discrete positions, the RH problems become singular. For the singular RH problems, we can transform it into regular RH problems by using the dressing method [33]. In the abstract, we can solve the solution of singular $\mathrm{RH}$ problems accurately by the Plemelj formula and dressing method [34]-[38]. However, when the reflection coefficient has multiple higher-order poles [39], the ansatz using the dressing matrix may become complex. Therefore, in the next section of the calculation, we will solve the RH problems directly without using the dressing matrix. 


\section{The RH problem with multiple higher-order poles}

In order to obtain the explicit solution of equation (3), in this section, we will consider the reflection-less case, i.e., $d(\lambda)=0(\lambda \in \mathbb{R})$. Since $c(\lambda)$ is analytic on the upper half plane, there are only finite zeros points and no singular point on the upper half plane. Different from other solutions of equations: $c(\lambda)$ has both simple zero points and higher-order zeros points. Therefore, we suppose $c(\lambda)$ and $c^{*}\left(\lambda^{*}\right)$ have higher order poles $\left\{\lambda_{j}, \operatorname{Im} \lambda_{j}>0\right\}_{j=1}^{N}$ and $\left\{\lambda_{j}^{*}, \operatorname{Im} \lambda_{j}^{*}<0\right\}_{j=1}^{N}$. That is, $c(\lambda)$ and $c^{*}\left(\lambda^{*}\right)$ are extended to

$$
\begin{aligned}
& c(\lambda)=\left(\lambda-\lambda_{1}\right)^{n_{1}}\left(\lambda-\lambda_{2}\right)^{n_{2}} \times \cdots \times\left(\lambda-\lambda_{N}\right)^{n_{N}} c_{0}(\lambda), \\
& c^{*}\left(\lambda^{*}\right)=\left(\lambda-\lambda_{1}^{*}\right)^{n_{1}}\left(\lambda-\lambda_{2}^{*}\right)^{n_{2}} \times \cdots \times\left(\lambda-\lambda_{N}^{*}\right)^{n_{N}} c_{0}^{*}\left(\lambda^{*}\right),
\end{aligned}
$$

where $c_{0}(\lambda) \neq 0$ for all $\lambda\left(\operatorname{Im} \lambda_{j}>0\right)$.

Assuming $\lambda=\lambda_{j}$ in the scattering relation (17), we can obtain that the Jost solution $\left[\psi_{-}\right]_{1}$ is directly proportional to $\left[\psi_{+}\right]_{2}$, where the relation is

$$
\left[\psi_{+}\right]_{2}\left(x, t, \lambda_{j}\right)=d\left(\lambda_{j}\right) e^{-2 i\left[\lambda_{j} x+\left(h \lambda_{j}+\lambda_{j}^{2}+16 \epsilon \lambda_{j}^{5}\right) t\right]}\left[\psi_{-}\right]_{1}\left(x, t, \lambda_{j}\right),
$$

and the symmetry condition (7) is also satisfied

$$
\left[\psi_{+}\right]_{1}\left(x, t, \lambda_{j}^{*}\right)=-d^{*}\left(\lambda_{j}^{*}\right) e^{2 i\left[\lambda_{j}^{*} x+\left(h \lambda_{j}^{*}+\lambda_{j}^{*^{2}}+16 \epsilon \lambda_{j}^{5}\right) t\right]}\left[\psi_{-}\right]_{2}\left(x, t, \lambda_{j}^{*}\right) .
$$

Combined with the definition of RH problems given in (21), we can obtain the following residual conditions

$$
\begin{aligned}
& \operatorname{Res}_{\lambda=\lambda_{j}}\left(\frac{\left[\psi_{+}\right]_{2}(x, t, \lambda)}{c(\lambda)}\right)=\operatorname{Res}_{\lambda=\lambda_{j}}\left(\frac{d(\lambda)}{c(\lambda)} e^{-2 i\left[\lambda x+\left(h \lambda+\lambda^{2}+16 \epsilon \lambda^{5}\right) t\right]}\left[\psi_{-}\right]_{1}(x, t, \lambda)\right), \\
& \operatorname{Res}_{\lambda=\lambda_{j}^{*}}\left(\frac{\left[\psi_{+}\right]_{1}\left(x, t, \lambda^{*}\right)}{c^{*}\left(\lambda^{*}\right)}\right)=\operatorname{Res}_{\lambda=\lambda_{j}^{*}}\left(\frac{d^{*}\left(\lambda^{*}\right)}{c^{*}\left(\lambda^{*}\right)} e^{2 i\left[\lambda x+\left(h \lambda+\lambda^{2}+16 \epsilon \lambda^{5}\right) t\right]}\left[\psi_{-}\right]_{2}(x, t, \lambda)\right),
\end{aligned}
$$

which are very useful for us to solve the solutions related to simple poles. When $c(\lambda)$ has only $N$ single zeros, the RH problem can be solved directly by the residue condition, and the formulae of N-order soliton solution can be obtained by (26). Among them, we can refer to $[30,33,40,41]$. However, the residual condition is not sufficient since $c(\lambda)$ has multiple higher order zeros points, so we need to consider the coefficients related to the much higher negative powers of $\lambda-\lambda_{j}$ and $\lambda-\lambda_{j}^{*}$. Here, we work on the simplest case first that $c(\lambda)$ has only one high-order zero point.

3.1 The RH problem with one higher-order pole

Let

$$
c(\lambda)=\left(\lambda-\lambda_{0}\right)^{N} c_{0}(\lambda),
$$

with $c_{0}(\lambda) \neq 0$ for all $\lambda\left(\operatorname{Im} \lambda_{j}>0\right)$. Using the Laurent series expansion, we can expand $r(\lambda)$ and $r^{*}\left(\lambda^{*}\right)$ as follows

$$
r(\lambda)=r_{0}(\lambda)+\sum_{n=1}^{N} \frac{r_{n}}{\left(\lambda-\lambda_{0}\right)^{n}}, \quad r^{*}\left(\lambda^{*}\right)=r_{0}^{*}\left(\lambda^{*}\right)+\sum_{n=1}^{N} \frac{r_{n}^{*}}{\left(\lambda-\lambda_{0}^{*}\right)^{n}},
$$


where $r_{n}(n=1,2, \cdots, N)$ are defined by

$$
r_{n}=\lim _{\lambda \rightarrow \lambda_{0}} \frac{1}{(N-n) !} \frac{\partial^{N-n}}{\partial \lambda^{N-n}}\left[\left(\lambda-\lambda_{0}\right)^{N} r(\lambda)\right], \quad n=1,2, \ldots, N,
$$

and $r_{0}(\lambda)$ is analytic for all $\lambda\left(\operatorname{Im} \lambda_{j}>0\right)$. In addition, through the definition of $\mathbf{M}_{11}(x, t, \lambda)$ given by $(21)$, we can conclude that $r(\lambda)=r_{0}^{*}(\lambda)$ is the N-order pole of $\mathbf{M}_{11}(x, t, \lambda)$, and $r(\lambda)=r_{0}(\lambda)$ is the $\mathrm{N}$-order pole of $\mathbf{M}_{12}(x, t, \lambda)$. According to property 2.3 , we set the normalization condition as

$$
\mathbf{M}_{11}(x, t, \lambda)=1+\sum_{m=1}^{N} \frac{R_{m}(x, t)}{\left(\lambda-\lambda_{0}^{*}\right)^{m}}, \quad \mathbf{M}_{12}(x, t, \lambda)=\sum_{m=1}^{N} \frac{W_{m}(x, t)}{\left(\lambda-\lambda_{0}\right)^{m}},
$$

where $R_{m}(x, t)$ and $W_{m}(x, t)$ are unknown functions to be determined. Once we obtain $R_{m}(x, t)$ and $W_{m}(x, t)(m=1,2, \ldots, N)$, we can find the solution $\mathbf{M}(x, t, \lambda)$ the singular $\mathrm{RH}$ problem. At the same time, we can also get the solution $q(x, t)$ of the original equation (3) from expression (26).

First of all, we can solve $R_{m}(x, t)$ and $W_{m}(x, t)(m=1,2, \ldots, N)$. On the basis of Taylor series expansion, we can obtain

$$
\begin{aligned}
& e^{-2 i\left[\lambda x+\left(h \lambda+\lambda^{2}+16 \epsilon \lambda^{5}\right) t\right]}=\sum_{s=0}^{+\infty} \zeta_{s}(x, t)\left(\lambda-\lambda_{0}\right)^{s}, \\
& e^{2 i\left[\lambda x+\left(h \lambda+\lambda^{2}+16 \epsilon \lambda^{5}\right) t\right]}=\sum_{s=0}^{+\infty} \zeta_{s}^{*}(x, t)\left(\lambda-\lambda_{0}^{*}\right)^{s}, \\
& \mathbf{M}_{11}(x, t, \lambda)=\sum_{s=0}^{+\infty} \mu_{s}(x, t)\left(\lambda-\lambda_{0}\right)^{s}, \\
& \mathbf{M}_{12}(x, t, \lambda)=\sum_{s=0}^{+\infty} \gamma_{s}(x, t)\left(\lambda-\lambda_{0}^{*}\right)^{s},
\end{aligned}
$$

where

$$
\begin{aligned}
& \zeta_{s}(x, t)=\lim _{\lambda \rightarrow \lambda_{0}} \frac{1}{s !} \frac{\partial^{s}}{\partial \lambda^{s}} e^{-2 i\left[\lambda x+\left(h \lambda+\lambda^{2}+16 \epsilon \lambda^{5}\right) t\right]}, \\
& \mu_{s}(x, t)=\lim _{\lambda \rightarrow \lambda_{0}} \frac{1}{s !} \frac{\partial^{s}}{\partial \lambda^{s}} \mathbf{M}_{11}(x, t, \lambda), \\
& v_{s}(x, t)=\lim _{\lambda \rightarrow \lambda_{0}} \frac{1}{s !} \frac{\partial^{s}}{\partial \lambda^{s}} \mathbf{M}_{12}(x, t, \lambda), \quad s=0,1,2, \ldots
\end{aligned}
$$

Combining the scattering relation given in (17) with the $\mathrm{RH}$ problem given in (21), and then through equating the corresponding coefficients of $\left(\lambda-\lambda_{0}\right)^{-m}$ and $\left(\lambda-\lambda_{0}^{*}\right)^{-m}$ respectively. $R_{m}(x, t)$ and $W_{m}(x, t)(m=1,2, \ldots, N)$ can be expressed by $\mu_{s}(x, t)$ and $v_{s}(x, t)(s=1,2, \ldots, N)$ as follows

$$
\begin{aligned}
& R_{m}(x, t)=-\sum_{n=m}^{N} \sum_{s=0}^{n-m} r_{n}^{*} \zeta_{n-m-s}^{*}(x, t) v_{s}(x, t), \\
& W_{m}(x, t)=\sum_{n=m}^{N} \sum_{s=0}^{n-m} r_{n} \zeta_{n-m-s}(x, t) \mu_{s}(x, t),
\end{aligned}
$$


with $m=1,2, \ldots, N$.

Remark 3.1. Among them, (37a) and (36b) are actually only a general expression of the residue condition (30a) and (30b). When $N=1$, the above relation with residue condition are still valid.

In fact, $\mu_{s}(x, t)$ and $v_{s}(x, t)(s=1,2, \ldots, N)$ can also be represented by $R_{m}(x, t)$ and $W_{m}(x, t)(m=1,2, \ldots, N)$. By reviewing the definitions of $\mu_{s}(x, t)$ and $v_{s}(x, t)$ given in (36b) and (36c), and substituting (34) into the definitions of $\mu_{s}(x, t)$ and $v_{s}(x, t)$, we can get

$$
\begin{aligned}
& v_{s}(x, t)=\sum_{m=1}^{N}\left(\begin{array}{c}
m+s-1 \\
s
\end{array}\right) \frac{(-1)^{s} W_{m}(x, t)}{\left(\lambda_{0}^{*}-\lambda_{0}\right)^{m+s}}, \quad s=0,1,2, \ldots, \\
& \mu_{s}(x, t)= \begin{cases}1+\sum_{m=1}^{N} \frac{R_{m}(x, t)}{\left(\lambda_{0}-\lambda_{0}^{*}\right)^{m}}, & s=0, \\
\sum_{m=1}^{N}\left(\begin{array}{c}
m+s-1 \\
s
\end{array}\right) \frac{(-1)^{s} R_{m}(x, t)}{\left(\lambda_{0}-\lambda_{0}^{*}\right)^{m+s}}, & s=1,2, \ldots\end{cases}
\end{aligned}
$$

Substituting (38a) and (38b) into (37a) and (37b), we get the following system

$$
\begin{aligned}
& R_{m}(x, t)=-\sum_{n=m}^{N} \sum_{s=0}^{n-m} \sum_{l=1}^{N}\left(\begin{array}{c}
l+s-1 \\
s
\end{array}\right) \frac{(-1)^{s} r_{n}^{*} f_{n-m-s}^{*}(x, t) W_{l}(x, t)}{\left(\lambda_{0}^{*}-\lambda_{0}\right)^{l+s}} \\
& W_{m}(x, t)=\sum_{n=m}^{N} r_{n} f_{n-m}(x, t)+\sum_{n=m}^{N} \sum_{s=0}^{n-m} \sum_{l=1}^{N}\left(\begin{array}{c}
l+s-1 \\
s
\end{array}\right) \frac{(-1)^{s} r_{n} f_{n-m-s}(x, t) W_{l}(x, t)}{\left(\lambda_{0}-\lambda_{0}^{*}\right)^{l+s}}
\end{aligned}
$$

At this point, we introduce

$$
\begin{aligned}
& |R\rangle=\left[\begin{array}{llll}
R_{1} & R_{2} & \cdots & R_{N}
\end{array}\right]^{T}, \quad|W\rangle=\left[\begin{array}{llll}
W_{1} & W_{2} & \cdots & W_{N}
\end{array}\right]^{T}, \\
& \Omega=\left[\Omega_{m l}\right]_{N \times N}=\left[-\sum_{n=m}^{N} \sum_{s=0}^{n-m}\left(\begin{array}{c}
l+s-1 \\
s
\end{array}\right) \frac{(-1)^{s} r_{n}^{*} f_{n-m-s}^{*}(x, t)}{\left(\lambda_{0}^{*}-\lambda_{0}\right)^{l+s}}\right]_{N \times N}, \\
& |\xi\rangle=\left[\begin{array}{llll}
\xi_{1} & \xi_{2} & \cdots & \xi_{N}
\end{array}\right]^{T}, \quad \xi_{m}=\sum_{n=m}^{N} r_{n} f_{n-m}(x, t), \quad m, l=1,2, \cdots, N,
\end{aligned}
$$

where superscript " $T$ " means transposition. Thus, we can rewrite (39a)and (39b) in the following form

$$
|R\rangle=\Omega|W\rangle, \quad|W\rangle=|\xi\rangle-\Omega^{*}|R\rangle .
$$

Then, by using Cramer's rule, $|R\rangle$ and $|W\rangle$ are easily solved as

$$
|R\rangle=\Omega\left(I+\Omega^{*} \Omega\right)^{-1}|\xi\rangle, \quad|W\rangle=\left(I+\Omega^{*} \Omega\right)^{-1}|\xi\rangle .
$$


Substituting $|R\rangle$ and $|W\rangle$ into expressions $\mathbf{M}_{11}(x, t, \lambda)$ and $\mathbf{M}_{12}(x, t, \lambda)$ given by (34), we can obtain

$$
\begin{aligned}
& \mathbf{M}_{11}(x, t, \lambda)=1+\sum_{m=1}^{N} \frac{R_{m}(x, t)}{\left(\lambda-\lambda_{0}^{*}\right)^{m}}=\frac{\operatorname{det}\left(I+\Omega^{*} \Omega+|\xi\rangle\langle Y(\lambda)| \Omega\right)}{\operatorname{det}\left(I+\Omega^{*} \Omega\right)}, \\
& \mathbf{M}_{12}(x, t, \lambda)=\sum_{m=1}^{N} \frac{G_{m}(x, t)}{\left(\lambda-\lambda_{0}\right)^{m}}=\frac{\operatorname{det}\left(I+\Omega^{*} \Omega+|\xi\rangle\left\langle Y^{*}\left(\lambda^{*}\right)\right|\right)}{\operatorname{det}\left(I+\Omega^{*} \Omega\right)}-1,
\end{aligned}
$$

where

$$
\langle Y(\lambda)|=\left[\begin{array}{llll}
\frac{1}{\left(\lambda-\lambda_{0}^{*}\right)} & \frac{1}{\left(\lambda-\lambda_{0}^{*}\right)^{2}} & \cdots & \frac{1}{\left(\lambda-\lambda_{0}^{*}\right)^{N}}
\end{array}\right] .
$$

In the symmetric property given by $(25)$, we obtain

$$
\begin{aligned}
& \mathbf{M}_{21}(x, t, \lambda)=1-\frac{\operatorname{det}\left(I+\Omega \Omega^{*}+\left|\xi^{*}\right\rangle\langle Y(\lambda)| \Omega\right)}{\operatorname{det}\left(I+\Omega \Omega^{*}\right)}, \\
& \mathbf{M}_{22}(x, t, \lambda)=\frac{\operatorname{det}\left(I+\Omega \Omega^{*}+\left|\xi^{*}\right\rangle\left\langle Y^{*}\left(\lambda^{*}\right)\right| \Omega\right)}{\operatorname{det}\left(I+\Omega \Omega^{*}\right)}-1 .
\end{aligned}
$$

On the basis of (26), we derive the following theorem

Theorem 3.2. Based on the rapidly decaying initial condition given by (11), the $\mathrm{n}$-th order BS soliton of the equation (3) is

$$
q(x, t)=2 i\left[\frac{\operatorname{det}\left(I+\Omega^{*} \Omega+|\xi\rangle\left\langle Y_{0}\right|\right)}{\operatorname{det}\left(I+\Omega^{*} \Omega\right)}-1\right]
$$

where $\left\langle Y_{0}\right|=\left[\begin{array}{lllll}1 & 0 & 0 & \cdots & 0\end{array}\right]_{1 \times N}$.

Proof. From the expression of $\mathbf{M}_{12}(x, t, \lambda)$ given in (43b), we get

$$
\begin{aligned}
q(x, t) & =\lim _{\lambda \rightarrow \infty} 2 i \lambda \mathbf{M}_{12}(x, t, \lambda) \\
& =\lim _{\lambda \rightarrow \infty} 2 i \lambda \times\left\langle Y^{*}\left(\lambda^{*}\right) \| W\right\rangle \\
& =\lim _{\lambda \rightarrow \infty} 2 i \lambda \times\left\langle Y^{*}\left(\lambda^{*}\right)\left|\left(I+\Omega^{*} \Omega\right)^{-1}\right| \xi\right\rangle \\
& =2 i \times\left\langle Y_{0}\left|\left(I+\Omega^{*} \Omega\right)^{-1}\right| \xi\right\rangle \\
& =2 i\left[\frac{\operatorname{det}\left(I+\Omega^{*} \Omega+|\xi\rangle\left\langle Y_{0}\right|\right)}{\operatorname{det}\left(I+\Omega^{*} \Omega\right)}-1\right] .
\end{aligned}
$$

The above theorem is proved.

Remark 3.3. If $N>1$, the BS solution of the equation can be obtained. Meanwhile, when $N=1$, we can also use expression (46) to get the first order soliton solution. 
3.2 RH problem with $\mathrm{N}$ higher-order poles

Now we will discuss the more general case that $c(\lambda)$ has $\mathrm{N}$ higher-order zeros points $\lambda_{1}, \lambda_{2}, \cdots, \lambda_{N} . \lambda_{1}, \lambda_{2}, \cdots, \lambda_{N}$ are on the upper half plane of the $\lambda$-plane, and their powers are $n_{1}, n_{2}, \cdots, n_{N}$ respectively. Similar to the case of the one higher-order pole discussed above, where we also use the Laurent series expansion, $r(\lambda)$ can be expressed as follows

$$
\begin{aligned}
& r(\lambda)=r_{0}(\lambda)+\frac{r_{1,1}}{\left(\lambda-\lambda_{1}\right)^{1}}+\frac{r_{1,2}}{\left(\lambda-\lambda_{1}\right)^{2}}+\cdots+\frac{r_{1, n_{1}}}{\left(\lambda-\lambda_{1}\right)^{n_{1}}} \\
&+ \frac{r_{2,1}}{\left(\lambda-\lambda_{2}\right)^{1}}+\frac{r_{2,2}}{\left(\lambda-\lambda_{2}\right)^{2}}+\cdots+\frac{r_{2, n_{2}}}{\left(\lambda-\lambda_{2}\right)^{n_{2}}} \\
& \vdots \\
&+ \frac{r_{N, 1}}{\left(\lambda-\lambda_{N}\right)^{1}}+\frac{r_{N, 2}}{\left(\lambda-\lambda_{N}\right)^{2}}+\cdots+\frac{r_{N, n_{N}}}{\left(\lambda-\lambda_{N}\right)^{n_{N}}} \\
&=r_{0}(\lambda)+\sum_{j=1}^{N} \sum_{m_{j}=1}^{n_{j}} \frac{r_{j, m_{j}}}{\left(\lambda-\lambda_{j}\right)^{m_{j}}},
\end{aligned}
$$

and

$$
r^{*}\left(\lambda^{*}\right)=r_{0}^{*}\left(\lambda^{*}\right)+\sum_{j=1}^{N} \sum_{m_{j}=1}^{n_{j}} \frac{r_{j, m_{j}}^{*}}{\left(\lambda-\lambda_{j}^{*}\right)^{m_{j}}},
$$

where

$$
r_{j, m_{j}}^{*}=\lim _{\lambda \rightarrow \lambda_{j}} \frac{1}{\left(n_{j}-m_{j}\right) !} \frac{\partial^{n_{j}-m_{j}}}{\left(\lambda-\lambda_{j}\right)^{n_{j}-m_{j}}}\left[\left(\lambda-\lambda_{j}\right)^{n_{j}} r(\lambda)\right]
$$

and $r_{0}(\lambda)$ is analytic for all $\lambda\left(\operatorname{Im} \lambda_{j}>0\right)$.

By using the similar method, we can obtain the following theorem

Theorem 3.4. If $c(\lambda)$ has $\mathrm{N}$-different high-order poles, we can obtain a result similar to that of expression (46) according to the rapidly initial condition of (11). Furthermore, the multiple BS solitons of the equation (3) is

$$
q(x, t)=2 i\left[\frac{\operatorname{det}\left(I+\Omega^{*} \Omega+|\xi\rangle\left\langle Y_{0}\right|\right)}{\operatorname{det}\left(I+\Omega^{*} \Omega\right)}-1\right],
$$

where

$$
\begin{aligned}
& |\xi\rangle=\left[\begin{array}{llll}
\xi_{1} & \xi_{2} & \cdots & \xi_{N}
\end{array}\right]^{T}, \\
& \left\langle Y_{0}\right|=\left[\begin{array}{llll}
Y_{1}^{0} & Y_{2}^{0} & \cdots & Y_{N}^{0}
\end{array}\right], \\
& \xi_{j}=\left[\begin{array}{llll}
\xi_{j, 1} & \xi_{j, 2} & \cdots & \xi_{j, n_{j}}
\end{array}\right], \\
& Y_{j}^{0}=\left[\begin{array}{llll}
1 & 0 & \cdots & 0
\end{array}\right]_{1 \times n_{j}} \text {, } \\
& \xi_{j, l}=\sum_{m_{j}=l}^{n_{j}} r_{j, m_{j}} f_{j, m_{j}-l}(x, t) \text {, } \\
& \Omega=\left[\begin{array}{cccc}
{\left[\omega_{11}\right]} & {\left[\omega_{12}\right]} & \cdots & {\left[\omega_{1 N}\right]} \\
{\left[\omega_{21}\right]} & {\left[\omega_{22}\right]} & \cdots & {\left[\omega_{2 N}\right]} \\
\vdots & \vdots & \ddots & \vdots \\
{\left[\omega_{N 1}\right]} & {\left[\omega_{N 2}\right]} & \cdots & {\left[\omega_{N N}\right]}
\end{array}\right]
\end{aligned}
$$


and $\left[\omega_{j, l}\right](j, l=1,2, \cdots, N)$ are $n_{j} \times n_{l}$ matrices with

$$
\left[\omega_{j, l}\right]=\left(\left[\omega_{j, l}\right]_{p, e}\right)_{n_{j} \times n_{l}}=\left(-\sum_{m_{j}=p}^{n_{j}} \sum_{s_{j}=0}^{m_{j}-p}\left(\begin{array}{c}
e+s_{j}-1 \\
s_{j}
\end{array}\right) \frac{(-1)^{s_{j}} r_{j, m_{j}}^{*} f_{j, m_{j}-p-s_{j}}^{*}(x, t)}{\left(\lambda_{j}^{*}-\lambda_{j}\right)^{s_{j}+e}}\right)_{n_{j} \times n_{l}} .
$$

Remark 3.5. When $c_{\lambda}$ has only one higher-order zero point, we can transform the formula of $q(x, t)$ expressed by (51) into the form of formula (46). Therefore, we get a special case that formula (46) is only (51).

From the above results, we can see that $\left[\omega_{j, l}\right]$ given by (53) is similar to $\Omega$ given by (40b). It can also be said that a block matrices of $\Omega$ composed of multiple higherorder poles (53) is constructed by a matrix $\Omega$ with one high-order pole $\Omega$ of (40b). Based on the above formula, we will find the solution of the equation (3) in the next section.

\section{The solutions of the equation (3)}

4.1 Solution related to two simple pole points

Let's first assume that $\lambda_{1}=a_{1}+i b_{1}$ and $\lambda_{2}=a_{2}+i b_{2}$ is one simple pole points of $c(\lambda)$, which is $r(\lambda)$, and we can write it in the following form

$$
r(\lambda)=r_{0}(\lambda)+\frac{r_{1,1}}{\lambda-\lambda_{1}}+\frac{r_{2,1}}{\lambda-\lambda_{2}} .
$$

According to Theorem 3.2, we get that $\Omega$ can be expressed as follows

$$
\begin{aligned}
\Omega_{1,1}=-\frac{r_{1,1}^{*} \zeta_{1,0}^{*}}{\lambda_{1}^{*}-\lambda_{1}}, & \Omega_{1,2}=-\frac{r_{1,1}^{*} \zeta_{1,0}^{*}}{\lambda_{1}^{*}-\lambda_{2}}, \\
\Omega_{2,1}=-\frac{r_{2,1}^{*} \zeta_{2,0}^{*}}{\lambda_{2}^{*}-\lambda_{1}}, & \Omega_{2,2}=-\frac{r_{2,1}^{*} \zeta_{2,0}^{*}}{\lambda_{2}^{*}-\lambda_{2}},
\end{aligned}
$$

$|\xi\rangle=\left[\begin{array}{ll}\xi_{1} & \xi_{2}\end{array}\right]$ are denoted as

$$
\xi_{1}=r_{1,1} \zeta_{1,0}(x, t), \quad \xi_{2}=r_{2,1} \zeta_{2,0}(x, t)
$$

and $\left\langle Y_{0}\right|=\left[\begin{array}{ll}1 & 1\end{array}\right]$. According to the expression (51), the explicit solution of the equation (3) can be obtained. We omit it here because of its explicit expression. In figure 1 , we mainly draw the image of zero boundary soliton with two pole points, which describes the role of two solitons in two different orbits. In figure 1(a), we draw a three-dimensional diagram of its, and figure 1(b) describes its density map. 


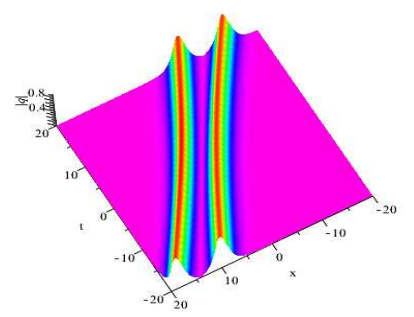

(a)

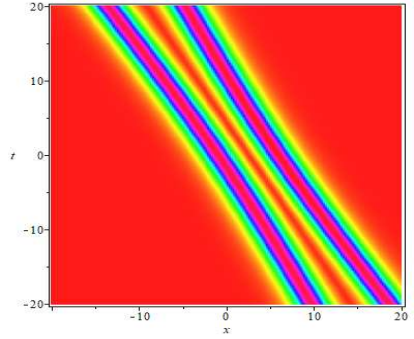

(b)

Figure 1. (Color online) The explicit solution of the equation (3) with $\lambda_{1}=\frac{1}{3}+\frac{\sqrt{3} i}{4}, \lambda_{2}=\frac{1}{4}+\frac{\sqrt{3} i}{4}$, $r_{1,1}=1, r_{2,1}=1, \epsilon=0, h=0$ : (a) The 3-dimensional plot. (b) The density plot.

4.2 Solution related to one second order pole

Let's first assume that $\lambda=\lambda_{0}$ is a second-order zero point of $c(\lambda)$, which is $r(\lambda)$, and we can write it in the following form

$$
r(\lambda)=r_{0}(\lambda)+\frac{r_{1}}{\lambda-\lambda_{0}}+\frac{r_{2}}{\left(\lambda-\lambda_{0}\right)^{2}}
$$

According to Theorem 3.2, we get that $\Omega$ can be expressed as follows

$$
\begin{aligned}
& \Omega_{11}=-\frac{r_{1}^{*} \zeta_{0}^{*}}{\lambda_{0}^{*}-\lambda_{0}}-\frac{r_{2}^{*} \zeta_{1}^{*}}{\lambda_{0}^{*}-\lambda_{0}}+\frac{r_{2}^{*} \zeta_{0}^{*}}{\left(\lambda_{0}^{*}-\lambda_{0}\right)^{2}}, \\
& \Omega_{12}=-\frac{r_{1}^{*} \zeta_{0}^{*}}{\left(\lambda_{0}^{*}-\lambda_{0}\right)^{2}}-\frac{r_{2}^{*} \zeta_{1}^{*}}{\left(\lambda_{0}^{*}-\lambda_{0}\right)^{2}}+\frac{2 r_{2}^{*} \zeta_{0}^{*}}{\left(\lambda_{0}^{*}-\lambda_{0}\right)^{3}}, \\
& \Omega_{21}=-\frac{r_{2}^{*} \zeta_{0}^{*}}{\lambda_{0}^{*}-\lambda_{0}}, \quad \Omega_{22}=-\frac{r_{2}^{*} \zeta_{0}^{*}}{\left(\lambda_{0}^{*}-\lambda_{0}\right)^{2}},
\end{aligned}
$$

$|\xi\rangle=\left[\begin{array}{ll}\xi_{1} & \xi_{2}\end{array}\right]^{T}$ are denoted as

$$
\xi_{1}=r_{1} \zeta_{0}+r_{2} \zeta_{1}, \quad \xi_{2}=r_{2} \zeta_{0}
$$

and $\left\langle Y_{0}\right|=\left[\begin{array}{ll}1 & 0\end{array}\right]$. According to the expression (51), the explicit solution of the equation (3) can be obtained. We omit it here because of its explicit expression. In order to better express the solution of the equation, we will describe it in the form of graph. In figure 2, we draw an image of the second-order BS soliton, which describes the staged interaction of two solitons in two curved orbits. In figure 2(a), we draw a three-dimensional diagram of the second-order BS soliton and figure 2(b) describes its density map. 


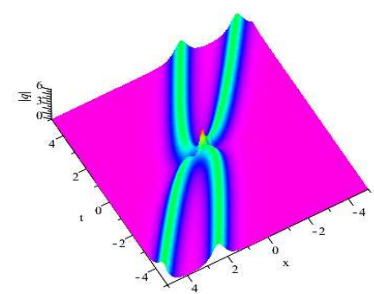

(a)

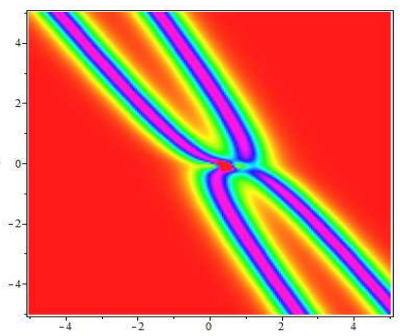

(b)

Figure 2. (Color online) The second-order BS soliton solution of the equation (3) with $\lambda_{0}=\frac{1}{3}+\sqrt{3} i$ and $r_{1}=1, r_{2}=2, \epsilon=0, h=0$ : (a) The 3-dimensional plot. (b) The density plot.

4.3 Solution related to one simple pole and one second-order pole

Let's first assume that $\lambda_{1}=a_{1}+i b_{1}$ is a simple zero point of $c(\lambda)$ and $\lambda_{2}=a_{2}+i b_{1}$ is one second-order pole points of $c(\lambda)$, which is $r(\lambda)$, and we can write it in the following form

$$
r(\lambda)=r_{0}(\lambda)+\frac{r_{1,1}}{\lambda-\lambda_{1}}+\frac{r_{1,2}}{\left(\lambda-\lambda_{1}\right)^{2}}++\frac{r_{2,1}}{\lambda-\lambda_{2}}
$$

According to Theorem 3.2, we get that $\Omega=\left[\begin{array}{l}{\left[\omega_{11}\right]\left[\omega_{12}\right]} \\ {\left[\omega_{21}\right]\left[\omega_{22}\right]}\end{array}\right]$ can be expressed as follows

$$
\begin{aligned}
& {\left[\omega_{11}\right]_{11}=-\frac{r_{1,1}^{*} \zeta_{1,0}^{*}}{\lambda_{1}^{*}-\lambda_{1}}-\frac{r_{1,2}^{*} \zeta_{1,1}^{*}}{\lambda_{1}^{*}-\lambda_{1}}+\frac{r_{1,2}^{*} \zeta_{1,0}^{*}}{\left(\lambda_{1}^{*}-\lambda_{1}\right)^{2}},} \\
& {\left[\omega_{11}\right]_{12}=-\frac{r_{1,1}^{*} \zeta_{1,0}^{*}}{\left(\lambda_{1}^{*}-\lambda_{1}\right)^{2}}-\frac{r_{1,2}^{*} \zeta_{1,1}^{*}}{\left(\lambda_{1}^{*}-\lambda_{1}\right)^{2}}+\frac{2 r_{1,2}^{*} \zeta_{1,0}^{*}}{\left(\lambda_{1}^{*}-\lambda_{1}\right)^{3}},} \\
& {\left[\omega_{11}\right]_{21}=-\frac{r_{1,2}^{*} \zeta_{1,0}^{*}}{\lambda_{1}^{*}-\lambda_{1}}, \quad\left[\omega_{11}\right]_{22}=-\frac{r_{1,2}^{*} \zeta_{1,0}^{*}}{\left(\lambda_{1}^{*}-\lambda_{1}\right)^{2}},} \\
& {\left[\omega_{12}\right]_{11}=-\frac{r_{1,1}^{*} \zeta_{1,0}^{*}}{\lambda_{1}^{*}-\lambda_{2}}-\frac{r_{1,2}^{*} \zeta_{1,1}^{*}}{\lambda_{1}^{*}-\lambda_{2}}+\frac{r_{1,2}^{*} \zeta_{1,0}^{*}}{\left(\lambda_{1}^{*}-\lambda_{2}\right)^{2}},} \\
& {\left[\omega_{12}\right]_{21}=-\frac{r_{1,2}^{*} \zeta_{1,0}^{*}}{\lambda_{1}^{*}-\lambda_{2}}, \quad\left[\omega_{21}\right]_{11}=-\frac{r_{2,1}^{*} \zeta_{2,0}^{*}}{\lambda_{2}^{*}-\lambda_{1}},} \\
& {\left[\omega_{21}\right]_{12}=-\frac{r_{2,1}^{*} \zeta_{2,0}^{*}}{\left(\lambda_{2}^{*}-\lambda_{1}\right)^{2}}, \quad\left[\omega_{22}\right]_{11}=-\frac{r_{2,1}^{*} \zeta_{2,0}^{*}}{\lambda_{2}^{*}-\lambda_{2}},}
\end{aligned}
$$

$|\xi\rangle=\left[\begin{array}{ll}\xi_{1} & \xi_{2}\end{array}\right]^{T}$ are denoted as

$$
\begin{aligned}
& \xi_{1,1}=r_{1,1} \zeta_{1,0}+r_{1,2} \zeta_{1,1}, \\
& \xi_{1,2}=r_{1,2} \zeta_{1,0}, \quad \xi_{2,1}=r_{2,1} \zeta_{2,0},
\end{aligned}
$$


and $\left\langle Y_{0}\right|=\left[\begin{array}{lll}1 & 0 & 1\end{array}\right]$. According to the expression (51), the explicit solution of the equation (3) can be obtained. We omit it here because of its explicit expression. In figure 3, we mainly describe the image of the interaction solution between one regular soliton and one second-order soliton. In figure 3(a), we can observe that the regular soliton passes through the center of the second-order BS soliton. After colliding with the branches of the two solitons, the original structure will be destroyed and oscillated periodically. In figure 3(c), the regular soliton collides with the left branch of the second-order BS soliton, and then the regular soliton oscillates periodically with its left branch, while the right branch remains unchanged. In figure 3(e), the regular soliton and the second-order BS soliton are separated immediately after the interaction, and keep the original shape, but there will be a certain displacement change. In figure 3(b), (d) and (f), we describe the density plots of their interactions respectively.

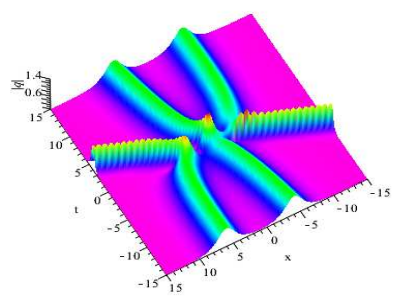

(a)

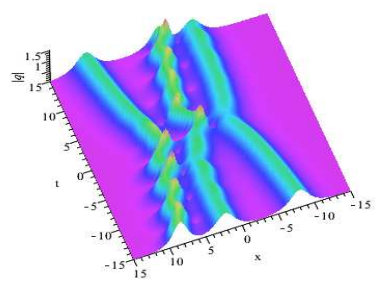

(c)

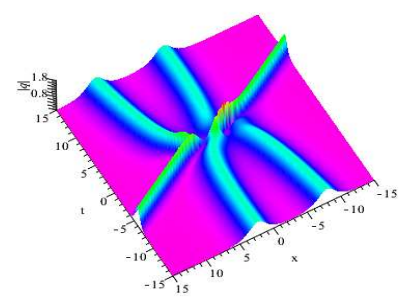

(e)

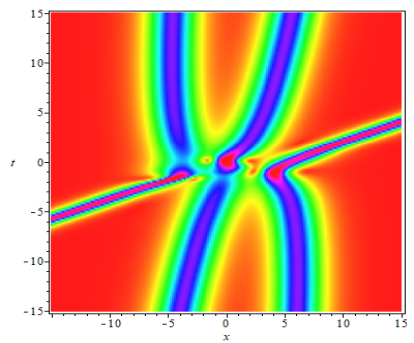

(b)

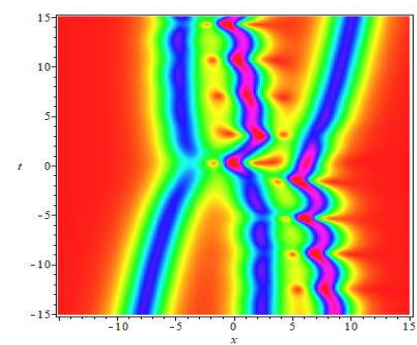

(d)

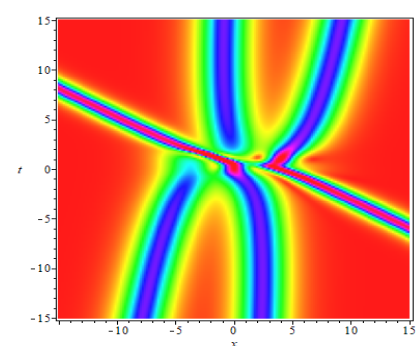

(f)

Figure 3. (Color online) The interaction between one soliton and one second-order BS solution of the equation (3) with $r_{1,1}=1, r_{1,2}=2, r_{2,1}=3, \epsilon=1, h=0$ : (a) The 3-dimensional plot with $\lambda_{1}=\frac{1}{5}+\frac{\sqrt{3} i}{4}$ and $\lambda_{2}=\frac{1}{3}+\frac{\sqrt{3} i}{3}$. (b) The density plot with $\lambda_{1}=\frac{1}{5}+\frac{\sqrt{3} i}{4}$ and $\lambda_{2}=\frac{1}{3}+\frac{\sqrt{3} i}{3}$. (c) The 3-dimensional plot 
with $\lambda_{1}=\frac{1}{5}+\frac{\sqrt{3} i}{4}$ and $\lambda_{2}=\frac{1}{5}+\frac{\sqrt{3} i}{3}$. (d) The density plot with $\lambda_{1}=\frac{1}{5}+\frac{\sqrt{3} i}{4}$ and $\lambda_{2}=\frac{1}{5}+\frac{\sqrt{3} i}{3}$. (e) The 3-dimensional plot with $\lambda_{1}=\frac{1}{5}+\frac{\sqrt{3} i}{4}$ and $\lambda_{2}=\frac{1}{20}+\frac{\sqrt{3} i}{3}$. (f) The density plot with $\lambda_{1}=\frac{1}{5}+\frac{\sqrt{3} i}{4}$ and $\lambda_{2}=\frac{1}{20}+\frac{\sqrt{3} i}{3}$.

\subsection{Solution related to two second-order pole points}

Let's first assume that $\lambda_{1}=a_{1}+i b_{1}$ and $\lambda_{2}=a_{2}+i b_{1}$ are two second-order pole points of $c(\lambda)$, which is $r(\lambda)$, and we can write it in the following form

$$
r(\lambda)=r_{0}(\lambda)+\frac{r_{1,1}}{\lambda-\lambda_{1}}+\frac{r_{1,2}}{\left(\lambda-\lambda_{1}\right)^{2}}+\frac{r_{2,1}}{\lambda-\lambda_{2}}+\frac{r_{2,2}}{\left(\lambda-\lambda_{2}\right)^{2}} .
$$

According to Theorem 3.2, we get that $\Omega=\left[\begin{array}{l}{\left[\omega_{11}\right]\left[\omega_{12}\right]} \\ {\left[\omega_{21}\right]\left[\omega_{22}\right]}\end{array}\right]$ can be expressed as follows

$$
\begin{aligned}
& {\left[\omega_{11}\right]_{11}=-\frac{r_{1,1}^{*} \zeta_{1,0}^{*}}{\lambda_{1}^{*}-\lambda_{1}}-\frac{r_{1,2}^{*} \zeta_{1,1}^{*}}{\lambda_{1}^{*}-\lambda_{1}}+\frac{r_{1,2}^{*} \zeta_{1,0}^{*}}{\left(\lambda_{1}^{*}-\lambda_{1}\right)^{2}},} \\
& {\left[\omega_{11}\right]_{12}=-\frac{r_{1,1}^{*} \zeta_{1,0}^{*}}{\left(\lambda_{1}^{*}-\lambda_{1}\right)^{2}}-\frac{r_{1,2}^{*} \zeta_{1,1}^{*}}{\left(\lambda_{1}^{*}-\lambda_{1}\right)^{2}}+\frac{2 r_{1,2}^{*} \zeta_{1,0}^{*}}{\left(\lambda_{1}^{*}-\lambda_{1}\right)^{3}},} \\
& {\left[\omega_{11}\right]_{21}=-\frac{r_{1,2}^{*} \zeta_{1,0}^{*}}{\lambda_{1}^{*}-\lambda_{1}}, \quad\left[\omega_{11}\right]_{22}=-\frac{r_{1,2}^{*} \zeta_{1,0}^{*}}{\left(\lambda_{1}^{*}-\lambda_{1}\right)^{2}},} \\
& {\left[\omega_{12}\right]_{11}=-\frac{r_{1,1}^{*} \zeta_{1,0}^{*}}{\lambda_{1}^{*}-\lambda_{2}}-\frac{r_{1,2}^{*} \zeta_{1,1}^{*}}{\lambda_{1}^{*}-\lambda_{2}}+\frac{r_{1,2}^{*} \zeta_{1,0}^{*}}{\left(\lambda_{1}^{*}-\lambda_{2}\right)^{2}},} \\
& {\left[\omega_{12}\right]_{12}=-\frac{r_{1,1}^{*} \zeta_{1,0}^{*}}{\left(\lambda_{1}^{*}-\lambda_{2}\right)^{2}}-\frac{r_{1,2}^{*} \zeta_{1,1}^{*}}{\left(\lambda_{1}^{*}-\lambda_{2}\right)^{2}}+\frac{2 r_{1,2}^{*} \zeta_{1,0}^{*}}{\left(\lambda_{1}^{*}-\lambda_{2}\right)^{3}},} \\
& {\left[\omega_{12}\right]_{21}=-\frac{r_{1,2}^{*} \zeta_{1,0}^{*}}{\lambda_{1}^{*}-\lambda_{2}}, \quad\left[\omega_{12}\right]_{22}=-\frac{r_{1,2}^{*} \zeta_{1,0}^{*}}{\left(\lambda_{1}^{*}-\lambda_{2}\right)^{2}},} \\
& {\left[\omega_{21}\right]_{11}=-\frac{r_{2,1}^{*} \zeta_{2,0}^{*}}{\lambda_{2}^{*}-\lambda_{1}}-\frac{r_{2,2}^{*} \zeta_{2,1}^{*}}{\lambda_{2}^{*}-\lambda_{1}}+\frac{r_{2,2}^{*} \zeta_{2,0}^{*}}{\left(\lambda_{2}^{*}-\lambda_{1}\right)^{2}},} \\
& {\left[\omega_{21}\right]_{12}=-\frac{r_{2,1}^{*} \zeta_{2,0}^{*}}{\left(\lambda_{2}^{*}-\lambda_{1}\right)^{2}}-\frac{r_{2,2}^{*} \zeta_{2,1}^{*}}{\left(\lambda_{2}^{*}-\lambda_{1}\right)^{2}}+\frac{2 r_{2,2}^{*} \zeta_{2,0}^{*}}{\left(\lambda_{2}^{*}-\lambda_{1}\right)^{3}},} \\
& {\left[\omega_{21}\right]_{21}=-\frac{r_{2,2}^{*} \zeta_{2,0}^{*}}{\lambda_{2}^{*}-\lambda_{1}}, \quad\left[\omega_{21}\right]_{22}=-\frac{r_{2,2}^{*} \zeta_{2,0}^{*}}{\left(\lambda_{2}^{*}-\lambda_{1}\right)^{2}},} \\
& {\left[\omega_{22}\right]_{11}=-\frac{r_{2,1}^{*} \zeta_{2,0}^{*}}{\lambda_{2}^{*}-\lambda_{2}}-\frac{r_{2,2}^{*} \zeta_{2,1}^{*}}{\lambda_{2}^{*}-\lambda_{2}}+\frac{r_{2,2}^{*} \zeta_{2,0}^{*}}{\left(\lambda_{2}^{*}-\lambda_{2}\right)^{2}},} \\
& {\left[\omega_{22}\right]_{12}=-\frac{r_{2,1}^{*} \zeta_{2,0}^{*}}{\left(\lambda_{2}^{*}-\lambda_{2}\right)^{2}}-\frac{r_{2,2}^{*} \zeta_{2,1}^{*}}{\left(\lambda_{2}^{*}-\lambda_{2}\right)^{2}}+\frac{2 r_{2,2}^{*} \zeta_{2,0}^{*}}{\left(\lambda_{2}^{*}-\lambda_{2}\right)^{3}} \text {, }} \\
& {\left[\omega_{22}\right]_{21}=-\frac{r_{2,2}^{*} \zeta_{2,0}^{*}}{\lambda_{2}^{*}-\lambda_{2}}, \quad\left[\omega_{22}\right]_{22}=-\frac{r_{2,2}^{*} \zeta_{2,0}^{*}}{\left(\lambda_{2}^{*}-\lambda_{2}\right)^{2}},}
\end{aligned}
$$


$|\xi\rangle=\left[\begin{array}{ll}\xi_{1} & \xi_{2}\end{array}\right]^{T}$ are denoted as

$$
\begin{array}{ll}
\xi_{1,1}=r_{1,1} \zeta_{1,0}+r_{1,2} \zeta_{1,1}, & \xi_{1,2}=r_{1,2} \zeta_{1,0}, \\
\xi_{2,1}=r_{2,1} \zeta_{2,0}+r_{2,2} \zeta_{2,1}, & \xi_{2,2}=r_{2,2} \zeta_{2,0},
\end{array}
$$

and $\left\langle Y_{0}\right|=\left[\begin{array}{llll}1 & 0 & 1 & 0\end{array}\right]$. According to the expression (51), the explicit solution of the equation (3) can be obtained. We omit it here because of its explicit expression. In figure 4, we describe the interaction of two second-order BS solitons. In figure 4(a), the two BS solitons evolve along two parallel orbits, and the interaction will cause the amplitude to oscillate periodically. In figure 4(c), the two BS solitons move at the same speed and shape. At the same time, their density maps are described in figure 4 (b) and (d), respectively.

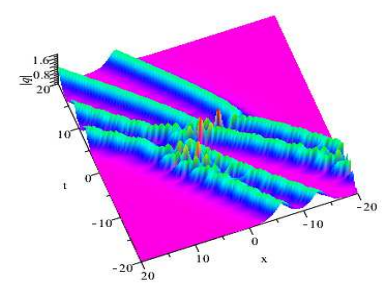

(c)

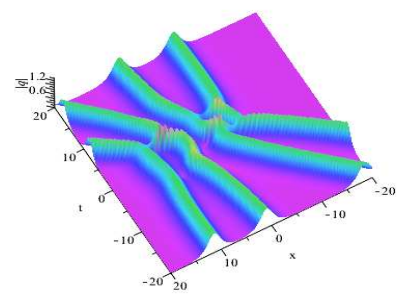

(c)

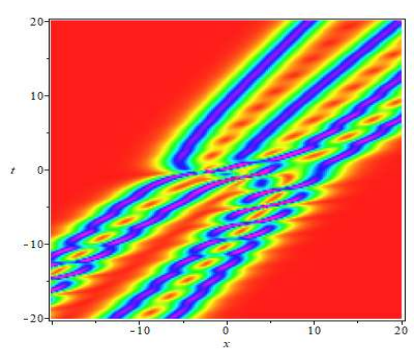

(b)

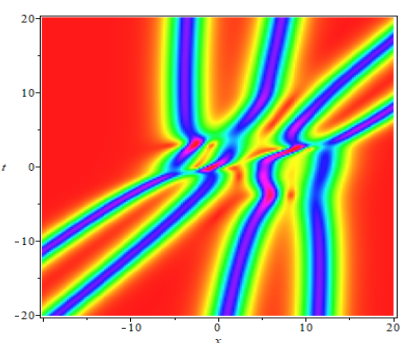

(d)

Figure 4. (Color online) The interaction between two second-order BS solution of the equation (3) with $r_{1,1}=1, r_{1,2}=5, r_{2,1}=5, r_{2,2}=1, \epsilon=1, h=0$ : (a) The 3-dimensional plot with $\lambda_{1}=\frac{1}{2}+\frac{\sqrt{3} i}{4}$ and $\lambda_{2}=\frac{1}{3}+\frac{\sqrt{3} i}{4}$. (b) The density plot with $\lambda_{1}=\frac{1}{2}+\frac{\sqrt{3} i}{4}$ and $\lambda_{2}=\frac{1}{3}+\frac{\sqrt{3} i}{4}$. (c) The 3-dimensional plot with $\lambda_{1}=\frac{1}{3}+\frac{\sqrt{3} i}{4}$ and $\lambda_{2}=\frac{1}{5}+\frac{\sqrt{3} i}{4}$. (d) The density plot with $\lambda_{1}=\frac{1}{3}+\frac{\sqrt{3} i}{4}$ and $\lambda_{2}=\frac{1}{5}+\frac{\sqrt{3} i}{4}$.

\section{Conclusions and discussions}

In this paper, we used the RH problem to study a higher-order nonlinear Schrödinger equation for inhomogeneous Heisenberg ferromagnetic system (3). We first obtained the Jost solution of lax pair, and constructed RH problem on the basis of Jost solution. Then, we solved the RH problem of scattering data in the case of one high-order pole and multiple high-order poles, and further derived the calculation formulas of BS solitons and multiple BS solitons in equation (3). In section 4, we obtained the 
simple order BS soliton, the second order BS soliton, and the interaction between the bound state soliton and the BS soliton in equation (3). In figure 3, by changing the corresponding parameter coefficients, one regular soliton and one second-order soliton will only had a certain displacement when they interact with each other, and their shapes will remain the original state. In figure 4, we can observed that the two BS solitons evolve along two parallel orbits, and the interaction will lead to periodic oscillation of amplitude, and they move at the same shape and speed.

Acknowledgements This work was supported by the National Natural Science Foundation of China under Grant No. 11975306, the Natural Science Foundation of Jiangsu Province under Grant No. BK20181351, the Six Talent Peaks Project in Jiangsu Province under Grant No. JY-059, and the Fundamental Research Fund for the Central Universities under the Grant Nos. 2019ZDPY07 and 2019QNA35.

\section{Compliance with ethical standards}

Conflict of Interest The authors declare that they have no conflict of interest.

\section{References}

1. K. Porsezian, K., Daniel, M., Lakshmanan, M.: On the integrability aspects of the one-dimensional classical continuum isotropic biquadratic Heisenberg spin chain, J. Math. Phys. 33, 1807 (1992).

2. Arnesen, M. C., Bose, S., Vedral, V.: Natural thermal and magnetic entanglement in the 1D Heisenberg model, Phys. Rev. Lett. 87, 017901 (2001).

3. Syljuäsen, O. F.: Entanglement and spontaneous symmetry breaking in quantum spin models, Phys. Rev. A 68, 060301(R) (2003).

4. Deng, D. L., Gu, S. J., Chen, J. L.: Detect genuine multipartite entanglement in the one-dimensional transverse-field Ising model, Annals Phys. 325(2), 367-372 ( 2010).

5. Asoudeh, M., Karimipour, V.: Thermal entanglement of spins in the Heisenberg model at low temperatures, Phys. Rev. A 70, 052307 (2004).

6. Lakshmanan, M.: Continuum spin system as an exactly solvable dynamical system, Phys. Lett. A 61(1), 53-54 (1977).

7. Zakharov, V. E., Takhtajan, L. A.: Equivalence of the nonlinear Schrödinger equation and the equation of a Heisenberg ferromagnet, Theor. Math. Phys. 38, 17-23 (1979).

8. Wazwaz, A. M., Xu, G. Q.: Kadomtsev-Petviashvili hierarchy: two integrable equations with timedependent coefficients. Nonlinear Dyn. 100, 3711-3716 (2020).

9. Wazwaz, A. M., Kaur, L.: New integrable Boussinesq equations of distinct dimensions with diverse variety of soliton solutions. Nonlinear Dyn. 97, 83-94 (2019)

10. Zhao, W. Z., Bai, Y. Q., Wu, K.: Generalized inhomogeneous Heisenberg ferromagnet model and generalized nonlinear Schrödinger equation, Phys. Lett. A 352(1-2), 64-68 (2006).

11. Zakharov, V. E., Shabat, A. B.: Exact theory of two-dimensional self-focusing and onedimensional self-modulation of waves in nonlinear media, Sov. Phys. JETP 34, 62-69 (1972).

12. Zakharov, V. E.: Stability of periodicwaves of finite amplitude on the surface of a deep fluid, J. Appl. Mech. Tech. Phys. 4, 190-194 (1968).

13. Lakshmanan, M.: Continuum spin system as an exactly solvable dynamical system, Phys. Lett. A 61(1), 53-54 (1977)

14. Jia, H. X., Ma, J. Y., Liu, Y. J., Liu, X. F.: Rogue-wave solutions of a higher-order nonlinear Schrödinger equation for inhomogeneous Heisenberg ferromagnetic system, Indian J. Phys. 89(3), 281287 (2015).

15. Conforti, M., Mussot, A., Kudlinski, A., Trillo, S., Akhmediev, N.: Doubly periodic solutions of the focusing nonlinear Schrödinger equation: Recurrence, period doubling, and amplification outside the conventional modulation-instability band, Phys. Rev. A 101, 023843 (2020).

16. Gardner, C. S., Greene, J. M., Kruskal, M. D., Miura, R. M.: Method for solving the Korteweg-de Vries equation, Phys. Rev. Lett. 19, 1095-1097 (1967). 
17. Gardner, C. S., Greene, J. M., Kruskal, M. D., Miura, R. M.: Korteweg-de Vries equation and generalizations. VI. methods for exact solution, Commun. Pur. Appl. Math. 27, 97-133 (1976).

18. Zhu, J. Y., Wang, L. L., Geng, X. G.: Riemann-Hilbert approach to TD equation with nonzero boundary condition, Front. Math. China 13, 1245-1265 (2018).

19. Geng, X., Wu, J.: Riemann-Hilbert approach and N-soliton solutions for a generalized Sasa-Satsuma equation. Wave. Motion. 60, 62-72 (2016).

20. Zhang, G. Q., Yan, Z. Y.: Inverse scattering transforms and soliton solutions of focusing and defocusing nonlocal mKdV equations with non-zero boundary conditions, Phys. D 402(15), 132170 (2020).

21. Wen, L. L., Fan, E. G.: The Sasa-Satsuma equation with non-vanishing boundary conditions, arXiv preprint arXiv:1911.11944 (2019).

22. Wang, X.B., Han, B.: Application of the Riemann-Hilbert method to the vector modified Korteweg-de Vries equation. Nonlinear Dyn. 99, 1363-1377 (2020)

23. Wang, J., Su, T., Geng, X. G., Li, R. M.: Riemann-Hilbert approach and N-soliton solutions for a new two-component Sasa-Satsuma equation. Nonlinear Dyn 101, 597-609 (2020).

24. Liu, N., Guo, B. L.: Solitons and rogue waves of the quartic nonlinear Schrö dinger equation by Riemann-Hilbert approach. Nonlinear Dyn. 100, 629-646 (2020)

25. Yan, X.W.: Riemann-Hilbert method and multi-soliton solutions of the Kundu-nonlinear Schrödinger equation. Nonlinear Dyn. 102, 2811-2819 (2020)

26. Guo, N., Xu, J., Wen, L. L., Fan, E.G.: Rogue wave and multi-pole solutions for the focusing KunduEckhaus Equation with nonzero background via Riemann-Hilbert problem method. Nonlinear Dyn https://doi.org/10.1007/s11071-021-06205-9

27. Yang, J. J., Tian, S. F., Li, Z. Q.: Inverse scattering transform and soliton solutions for the modified matrix Korteweg-de Vries equation with nonzero boundary conditions, arXiv:2005.00290v1 (2020).

28. Bilman, D., Miller, P. D.: A robust inverse scattering transform for the focusing nonlinear Schrödinger equation, Commun. Pur. Appl. Math. 72, 1722-1805 (2019).

29. Bilman, D., Buckingham, R.: Large-order asymptotics for multiple-pole solitons of the focusing nonlinear Schrödinger equation, J. Nonlinear Sci. 29, 2185-2229 (2019).

30. Ablowitz, M. J., Clarkson, P. A.: Solitons, nonlinear evolution equations and inverse scattering, Cambridge university press, Cambridge, (1991).

31. Ablowitz, M. J., Prinari, B., Trubatch, A.: Discrete and continuous nonlinear Schrödinger systems, Cambridge University Press, Cambridge, (2004).

32. Ablowitz, M., Fokas, A.: Introduction and applications of complex variables, Cambridge University Press, Cambridge, (2003).

33. Novikov, S., Manakov, S., Pitaevskii, L., Zakharov, V.: Theory of solitons: the inverse scattering method, Consultants Bureau, New York, (1984).

34. Gagnon, L., Stiévenart, N.: N-soliton interaction in optical fibers: the multiple-pole case, Opt. Lett. 19, 619-621 (1994).

35. Shchesnovich, V. S., Yang, J.: General soliton matrices in the Riemann-Hilbert problem for integrable nonlinear equations, J. Math. Phys. 44, 4604-4639 (2003).

36. Wang, D. S., Zhang, D. J., Yang, J.: Integrable properties of the general coupled nonlinear Schrödinger equations, J. Math. Phys. 51, 023510 (2010).

37. Yang, J.: Nonlinear waves in integrable and non-integrable systems, SIAM, Philadelphia, (2010).

38. Huang, X., Guo, B., Ling, L.: Darboux transformation and novel solutions for the long wave-short wave model, J. Nonlinear Math. Phys. 20, 514-528 (2013).

39. Yang, J., Zhu, J., Wang, L.: Dressing by regularization to the Gerdjikov-Ivanov equation and the higher-order soliton, arXiv:1504.03407.

40. Faddeev, L. D., Takhtajan, L. A.: Hamiltonian methods in the theory of solitons, Springer, Berlin, (1987).

41. Zhang, Y. S., Tao, X. X., Xu, S. W.: The bound-state soliton solutiond of the complex modified KdV equation, Inverse Problems, (2020) 


\section{Figures}

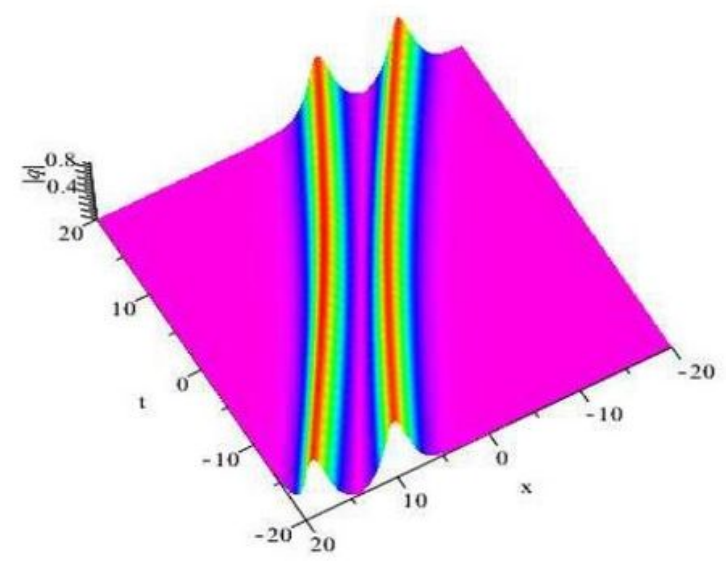

(a)

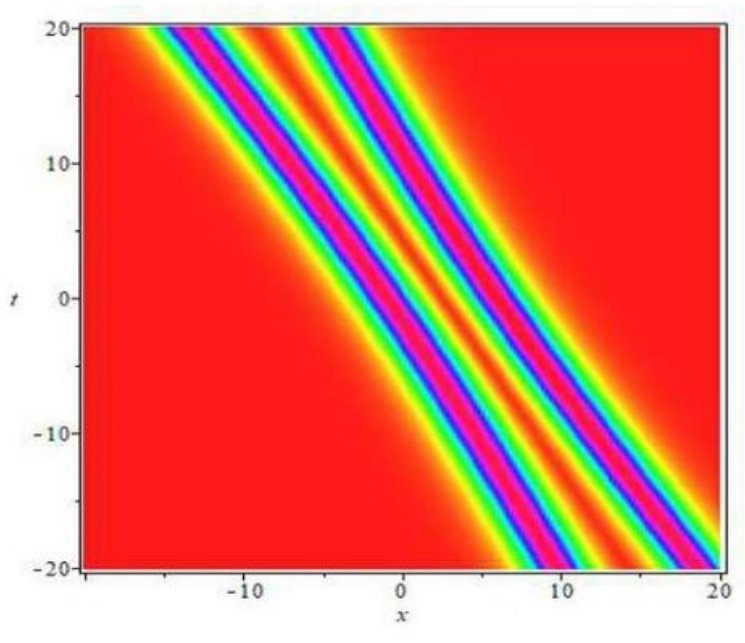

(b)

\section{Figure 1}

Please see the Manuscript PDF file for the complete figure caption

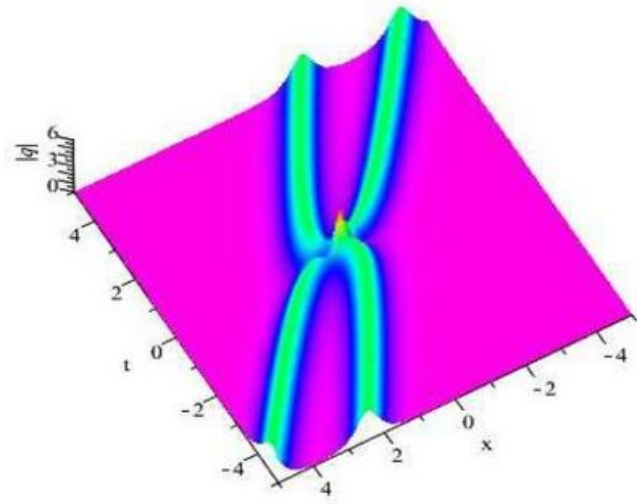

(a)

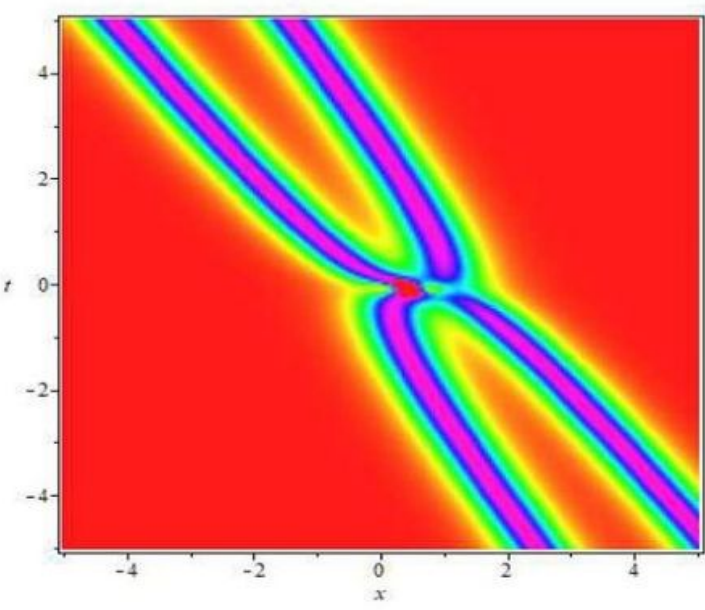

(b)

Figure 2

Please see the Manuscript PDF file for the complete figure caption 


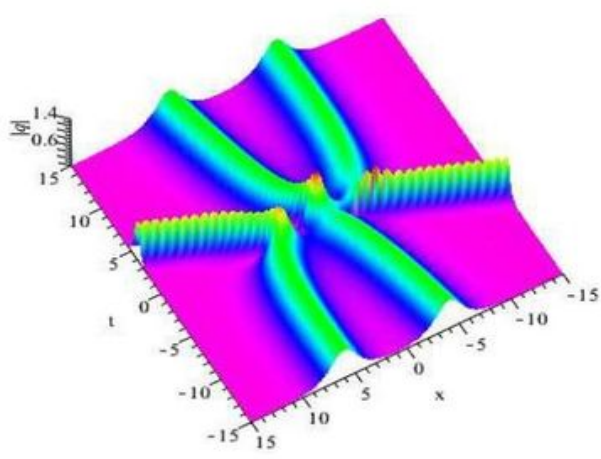

(a)

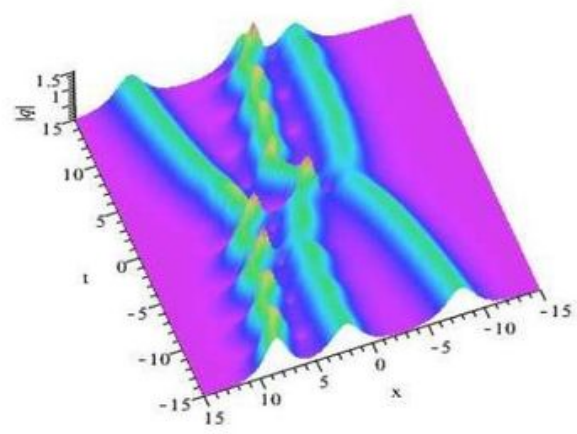

(c)

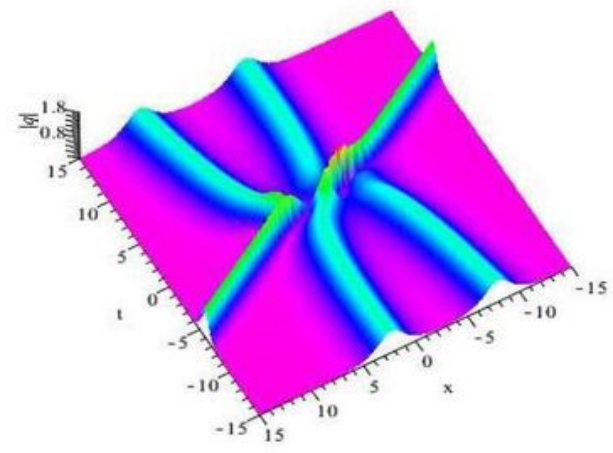

(e)

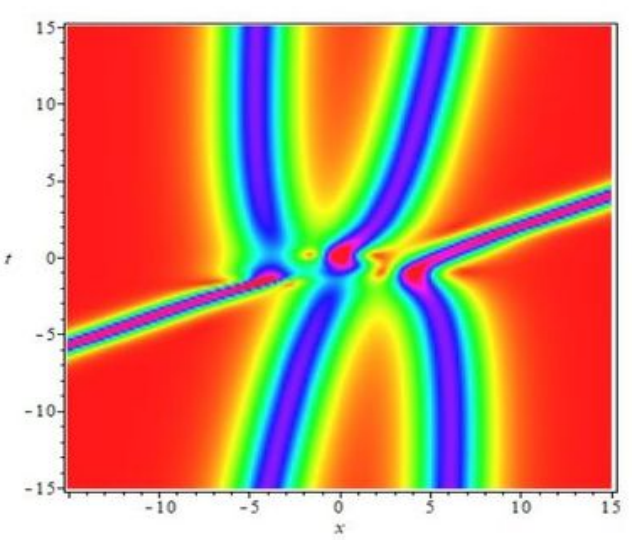

(b)

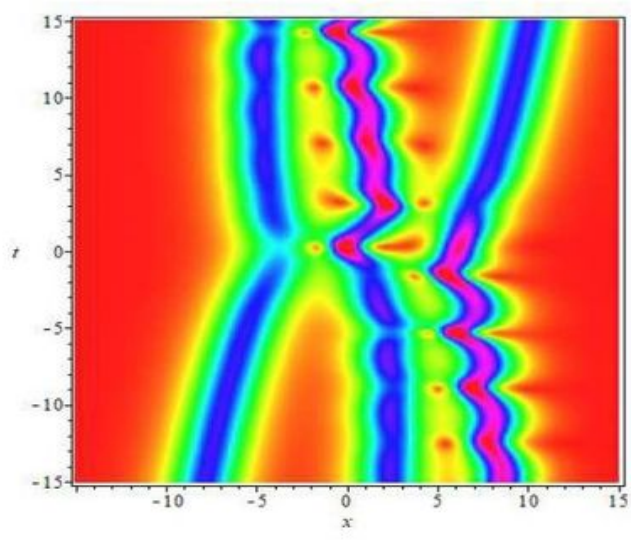

(d)

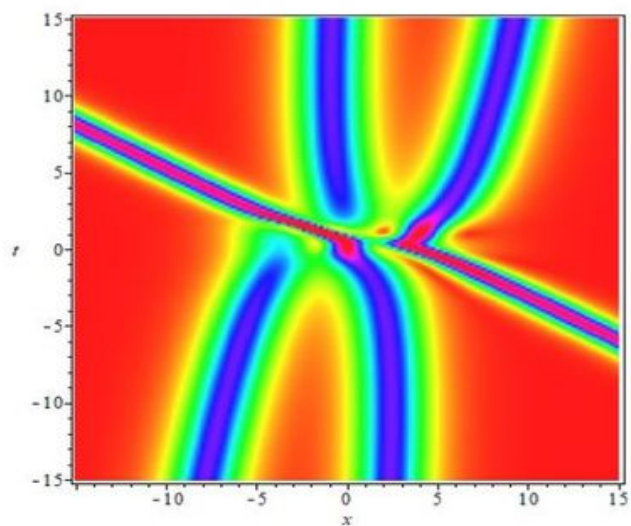

(f)

Figure 3

Please see the Manuscript PDF file for the complete figure caption 


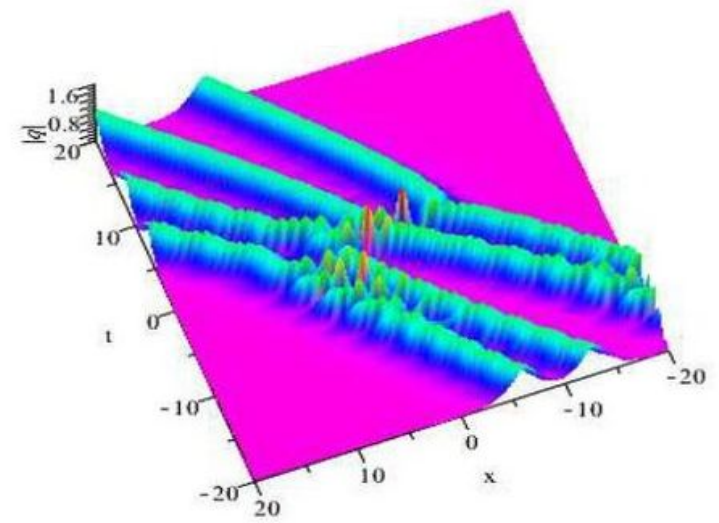

(c)

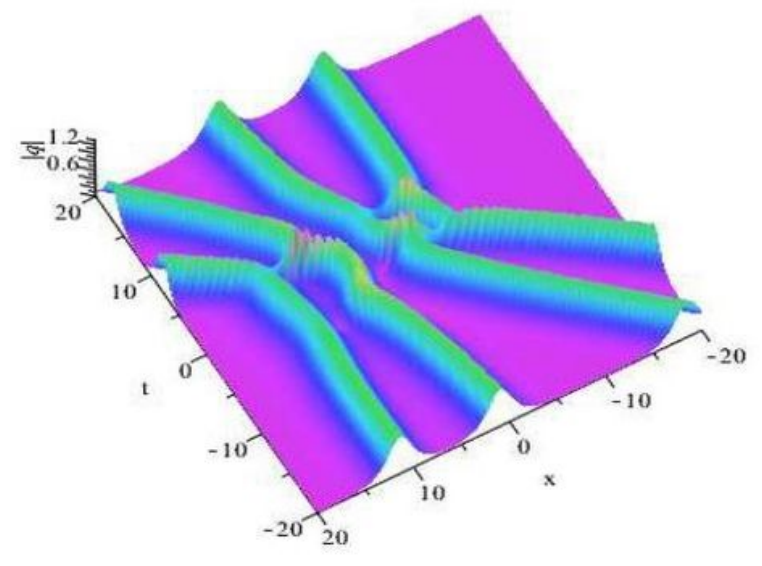

(c)

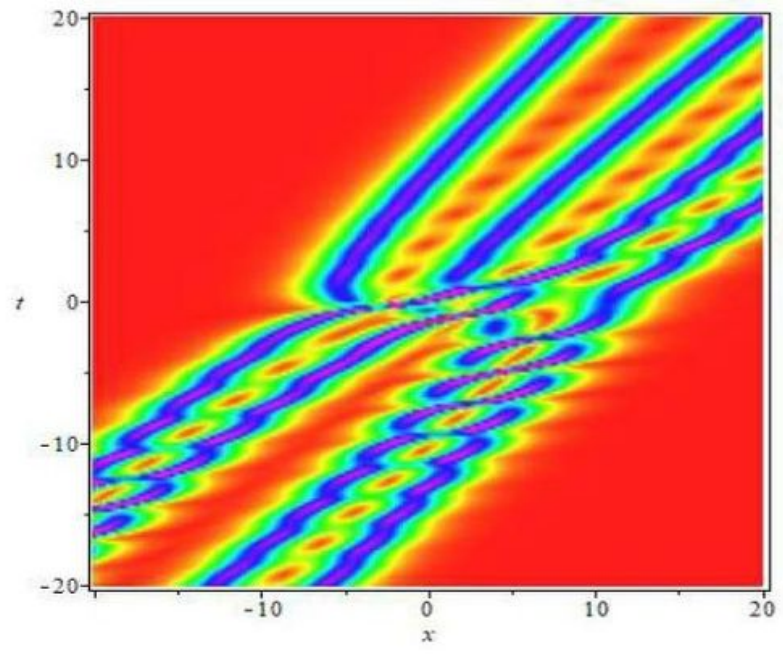

(b)

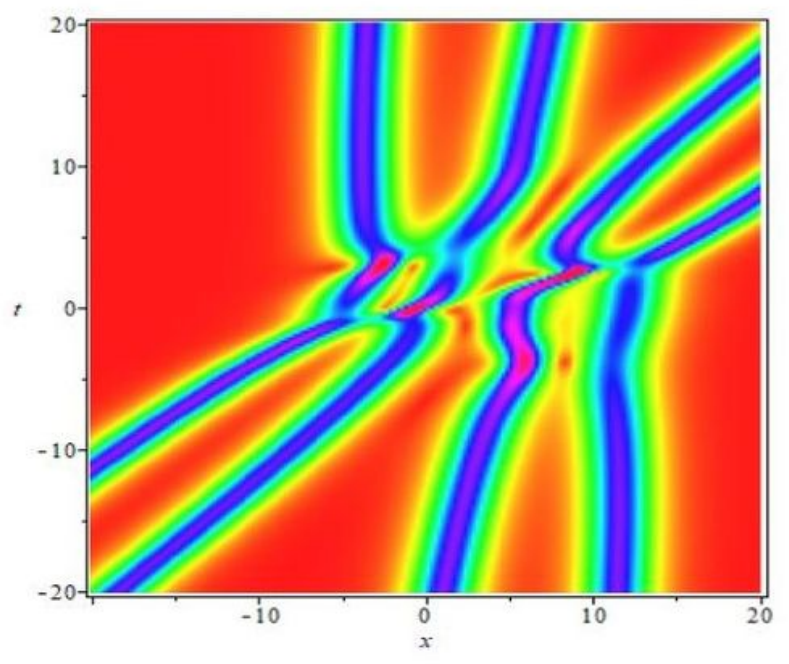

(d)

Figure 4

Please see the Manuscript PDF file for the complete figure caption 